\title{
Relationship Between Infertility and Digital Cervicography Findings in Cameroonian Women
}

\author{
Simon M. Manga ${ }^{1,2^{*}}$, Yan $\mathrm{Li}^{3}$, Kathleen L. Nulah², Mireille Kanjo ${ }^{2}$, Edith R. Welty ${ }^{2}$ Comfort Enah ${ }^{4}$, \\ Alan T. Tita ${ }^{5}$
}

\begin{abstract}
Objectives: Cervical neoplasia and some forms of infertility are caused by sexually transmitted infections. We aimed at evaluating whether positive findings on digital cervicography (DC) (performed to screen and treat cervical neoplasia) is associated with female infertility.

Materials and Methods: In this cross-sectional study, women consulting for infertility underwent cervical cancer screening using DC alongside women without infertility. A total of 2128 Cameroonian women were studied. Women with infertility were subcategorized into primary and secondary infertility. Potential confounders were assessed at the screening visit and logistic regression was used to compute adjusted relative risks for the relationship between infertility and positive DC findings.

Results: Among 2128 women studied, 292 (13.7\%) presented due to infertility and 1836 did not; 88 (30.1\%) of women with infertility had primary infertility and $204(69.9 \%)$ had secondary infertility. Women with infertility had a positive DC prevalence rate of $15.9 \%$ compared to $13.6 \%$ for women without infertility $(P=0.31)$. The prevalence rates of positive DC among women with primary infertility or among those with secondary infertility respectively, did not differ from the rate in those without infertility. After multivariable adjustments relative to those without infertility, the adjusted relative risks (aRRs) (95\% CI) for a positive DC were: 0.91 (0.62-1.20) for any infertility, $0.99(0.65-1.51)$ for secondary infertility and $0.73(0.38-1.41)$ for primary infertility.

Conclusions: Results of DC were not significantly associated with infertility (and its subtypes). Further investigations are required to evaluate the association with other cervical cancer screening modalities like Pap smear and HPV DNA-testing.

Keywords: Cervical neoplasia, Cervical cancer screening, Digital Cervicography, Primary infertility, Secondary infertility
\end{abstract}

\section{Introduction}

The relationship between cervical neoplasia and female infertility has not been properly explored. Cervical neoplasia is associated with a sexually transmitted infection (STI) and some form of female infertility is also associated with STIs (1). Because there is high probability for STIs to occur concurrently $(2,3)$, there is a likelihood for an association between conditions caused by STIs such as those between cervical neoplasia and female infertility. The purpose of this investigation was to find out whether cervical neoplasia diagnosed by digital cervicography (DC) was associated with female infertility among Cameroonian women.

Cancer of the cervix is the primary etiology of cancer morbidity and mortality in females in sub-Saharan Africa (SSA), where about 99000 new cases and 57400 deaths happened in $2012(4,5)$. It is the second leading cause of cancer death in females in Cameroon. It has an agestandardized incidence and mortality rates per 100000 females of 30.0 and 17.5 , respectively (4).

The principal cause of cervical neoplasia including precancerous and cancerous lesions, is high risk human papilloma virus (hrHPV) infection which happens to be among the most common STIs in the world (6). In addition, there are several potential risk factors connected with the development of cervical neoplasia such as early initiation of sexual intercourse, numerous sexual partners or a partner with numerous sexual partners, coinfection with human immune deficiency virus (HIV) and other STIs $(6,7)$. Current World Health Organization (WHO) guidelines for "screen-and-treat" programs for low- and middle-income countries (LMIC) endorse visual inspection with acetic acid (VIA) for primary screening to detect cervical neoplasia, if infrastructure for other screening modalities such as Pap smear and HPV testing does not exist, and underscore the importance of immediate treatment when possible, to assure that women with precancerous lesions are not lost to follow-up $(6,8)$.

Infertility is a condition that affects the reproductive system characterized by the inability to achieve a clinical gestation after one year or more of frequent unprotected penile-vaginal intercourse (9). There are two subtypes of infertility; primary infertility if the woman has never achieved a pregnancy in the past and secondary infertility

Received 15 December 2017, Accepted 10 March 2018, Available online 23 March 2018

${ }^{1}$ College of Nursing and Health Sciences, University of Massachusetts Boston, USA. ${ }^{2}$ Cameroon Baptist Convention Health Services, Women's Health Program, Bamenda, Cameroon. ${ }^{3}$ Department of Biostatistics, University of Alabama at Birmingham, USA. ${ }^{4}$ School of Nursing, University of Alabama at Birmingham, USA. ${ }^{5}$ Department of Obstetrics and Gynecology and Center for Women's Reproductive Health, University of Alabama at Birmingham, USA.

*Corresponding Author: Simon Manga, PhD Student; Tel: +1 7816006119, Email: Simon.Manga001@umb.edu 
if she has (10). Tubal occlusion which is associated with STIs is one of the most popular causes of female factor infertility $(1,11)$.

Since STIs are a major cause of infertility and sexual transmission of HPV is the main cause of cervical neoplasia, it is plausible that infertility may be associated with cervical neoplasia. While some studies have examined whether treatments of cervical neoplasia such as cervical excision or cryotherapy are associated with infertility $(6,12,13)$, our preliminary literature search of the entire PubMed database did not yield any recent study specifically examining the relationship between findings on cervical neoplasia screening and infertility. Therefore, we conducted this analysis to explore the relationship between female infertility (and its subtypes) and screening results for cervical neoplasia using a screening method known as DC. Our central hypothesis was that there is an association between infertility and positive (abnormal) cervical neoplasia results on DC.

\section{Materials and Methods}

Study Design and Setting

We implemented a cross-sectional study involving patients undergoing cervical cancer screening at the EtougEbe Baptist Hospital Yaoundé (EBHY), a facility of the Cameroon Baptist Convention Health Services (CBCHS), Cameroon. CBCHS is a faith-based organization that runs a system of 81 health facilities located in six of the 10 regions of Cameroon (http://www.cbchealthservices.org). CBCHS has a nurse-directed Women's Health Program (WHP) that has used DC to screen women for cervical cancer for the past 10 years according to WHO guidelines. DC involves the use of a digital camera or a Samsung cell phone camera to provide real-time images and permanent photographs of the acetic acid and Lugol's iodine stained cervix to determine whether a precancerous or cancerous lesion is present (14). WHP has a database where cervical photos (cervicographs) taken are linked to each woman's medical record. Cervicographs are stored and used for comparison during follow-up, capacity building, consultation, and quality improvement. WHP activities at EBHY facility also include consultations and workups for couples with infertility. All the women consulting for infertility are encouraged to do cervical cancer screening.

Study Population, Variable Ascertainment and Definitions All women seen at EBHY from January 2012 to December 2014, and who were screened for cervical cancer were eligible. Using the indications for their consultations and their medical history, we categorized participants regarding whether they had infertility or not. Infertility was defined as inability to archive a pregnancy for at least one year despite frequent unprotected penile-vaginal intercourse based on history; women without a prior pregnancy were considered to have primary infertility while those with prior pregnancy were considered to have secondary infertility.

For cervical cancer screening, the nurse took a cervical photo after insertion of a vaginal speculum. Thereafter, a $5 \%$ solution of acetic acid was applied on the cervix and a second cervical photo was taken after two minutes. The third cervical photo was taken immediately after the application of 5\% Lugol's iodine. After removal of the speculum, a bimanual exam was performed to assess for pelvic masses and pelvic inflammatory disease (PID). The findings of the DC were reported as positive, negative, inadequate, or uncertain. A positive result was defined as the presence of acetowhite lesion on DC. Results of Lugol's iodine were not used in this study, because a prior study of the WHP patient population had shown no significant difference between findings of visual inspection with acetic acid and those with Lugol's iodine (15). Of note, positive findings on DC correlate with precancerous or cancerous cervical neoplasia findings on pathology. A negative DC result was defined as the absence of acetowhite lesion after application of acetic acid or absence of leukoplakia. Inadequate result was defined as a negative result but where the transformation zone was not completely visible. An uncertain result was defined as a result in which a conclusion was difficult to make most probably due to the presence of cervicitis. For this report, we categorized cervical neoplasia as positive DC results and all the other DC results (negative, inadequate and uncertain) were included in the "no cervical neoplasia" or negative group. Women with cervical findings positive for precancerous lesions were treated either with cryotherapy, thermal coagulation or loop electric excision procedure (LEEP) and the women were given a one year follow up appointment; those with findings suspicious for invasive cancer or lesions that were difficult to interpret received same-day biopsies which were sent for histopathology at a specialized facility. Those with findings consistent with infections such as vaginitis, cervicitis and PID received appropriate antifungal or antibiotic therapy.

All women presenting for cervical cancer screening signed an informed consent at the time of screening. The consent form included statements indicating that their cervical photos (cervicographs) and other information could be used for teaching and scientific publications.

\section{Data Analysis}

Data analysis was performed using SAS 9.3 (SAS Institute, Cary, NC, USA). We assessed for associations between potential risk factors for infertility (co-variables) and DC findings. Next, we implemented univariable comparisons of DC results and other potential risk factors for infertility by infertility status. The potential risk factors considered are shown in Table 1. Chi-square or Fisher exact test were used as appropriate. Co-variables (except sex for money due to very small sample size) were used to fit logistic regressions for infertility (and sub-types). Crude and adjusted relative risk - RR (95\% CI) relating infertility 
Table 1. Characteristics of Women by DC Results

\begin{tabular}{|c|c|c|c|}
\hline \multirow[b]{2}{*}{ Characteristic } & \multicolumn{2}{|c|}{ DC Results } & \multirow[t]{2}{*}{$P$ Value } \\
\hline & $\begin{array}{c}\text { Positive } \\
\text { No. (\%) }\end{array}$ & $\begin{array}{c}\text { Negative } \\
\text { No. (\%) }\end{array}$ & \\
\hline Age group $(n=2104)$ & & & 0.46 \\
\hline $15-24$ & $54(18.4)$ & $317(17.5)$ & \\
\hline $25-34$ & $151(51.4)$ & $878(48.5)$ & \\
\hline $35+$ & $89(30.3)$ & $615(34.0)$ & \\
\hline Years of school $(n=2108)$ & & & 0.78 \\
\hline $0-7$ & $47(16.0)$ & $334(18.4)$ & \\
\hline $8-14$ & $70(23.8)$ & $425(23.4)$ & \\
\hline $15+$ & $49(16.7)$ & $301(16.6)$ & \\
\hline Unknown & $128(43.5)$ & $754(41.6)$ & \\
\hline Marital Status ${ }^{a}(n=2087)$ & & & 0.55 \\
\hline Married & $209(71.6)$ & $1264(70.4)$ & \\
\hline Single, not living with a man & $70(24.0)$ & $422(23.5)$ & \\
\hline Divorced/Separated/Widowed & $13(4.5)$ & $109(6.1)$ & \\
\hline Lifetime partners $(n=2108)$ & & & 0.12 \\
\hline 0-2 partners & $41(14.0)$ & 361 (19.9) & \\
\hline 3-5 partners & $72(24.5)$ & $412(22.7)$ & \\
\hline $6+$ partners & $14(4.8)$ & $87(4.8)$ & \\
\hline Unknown & $167(56.8)$ & $954(52.6)$ & \\
\hline Current RTI (n=2107) & & & 0.93 \\
\hline cervicitis/PID /vaginitis & $22(7.5)$ & $133(7.3)$ & \\
\hline Age at first sex $(n=1503)$ & & & 0.2 \\
\hline $0-14$ & $19(9.6)$ & $93(7.1)$ & \\
\hline $15-18$ & $118(59.3)$ & $730(56.0)$ & \\
\hline $19+$ & $62(31.2)$ & 481 (36.9) & \\
\hline Forced sex $(n=1937)$ & & & 0.43 \\
\hline Yes & $14(5.3)$ & $111(6.6)$ & \\
\hline Sex for money $(n=1925)$ & & & 0.37 \\
\hline Yes & $6(2.3)$ & $26(1.6)$ & \\
\hline
\end{tabular}

Abbreviations: DC, digital cervicography; RTI, Reproductive tract infection.

${ }^{a}$ Compared to the non-infertility group.

Note: The numbers in the tables may differ from the total number of participants because there are missing values for each variable, reason why the total $\mathrm{N}$ for each variable was provided.

status to DC results and other co-variable factors are provided. We chose to compute RR (a prevalence ratio) instead of OR due to the fact that the rate of infertility among women with negative DC result was $13.5 \%$ which cannot be considered as rare and OR will overestimate the association with infertility.

\section{Results}

From January 2012 to December 2014, EBHY Women's Health Program screened a total of 2128 women for cervical cancer. Out of these, $292(13.7 \%)$ were due to infertility while 1836 were not. The co-variable characteristics of the women who participated in the study by their DC results are shown in Table 1. Women with and without positive DC results did not vary significantly in any of the 8 features or co-variables examined.

The prevalence of positive DC findings (15.9\%) in those with infertility was similar to the prevalence in those without infertility (13.6\%), $P=0.31$ (Table 2). Not shown, DC results were negative in 74.5 vs. $77.6 \%$, inadequate in 9.2 vs. $5.5 \%$ and uncertain in 2.7 vs. $1.2 \%$ respectively in those with infertility vs. those without infertility. Further, women with infertility, when compared to those without infertility, were significantly more likely to be younger, report their educational status, be married and report the number of lifetime partners (Table 2). Both groups with and without infertility were comparable in the other covariables including a current diagnosis of STI (Table 2).

The unadjusted and adjusted results for the association between infertility and cervical neoplasia based on DC results and for other co-variables are presented in Table 3. Infertility overall was not significantly associated with the presence of cervical neoplasia (aRR, 0.91, 95\% CI: 0.621.20). Age 35 years or older and unmarried status were both independently associated with a reduced likelihood of infertility.

When further stratified by type of infertility in additional analyses, those with secondary infertility had a nonsignificantly higher prevalence of positive DC findings for cervical neoplasia than those with primary infertility; $18.2 \%$ vs. $10.3 \%, P=0.09$. After multivariable adjustments relative to those without infertility, neither primary infertility $(\mathrm{aRR}=0.73 ; 0.38-1.41)$ nor secondary infertility $(\mathrm{aRR}=0.99 ; 0.65-1.51)$ was significantly associated with cervical neoplasia. Both primary and secondary sub-types of infertility were less prevalent in those older than 35 years (respective aRRs, 0.23 [0.11-0.48] and 0.62 [0.42 $0.91]$ ) and those who were not currently married (aRRs $0.23[0.12-0.44]$ and $0.34[0.21-0.54])$. Participants that did not report years of schooling also had significantly lower prevalence of infertility.

\section{Discussion}

This is one of the very few published studies to evaluate the association between cervical cancer screening results and infertility. A search of the full PubMed database through March 2018 did not yield any recent study that examined the relationship between presence or absence of cervical neoplasia (by any screening method) and infertility. Contrary to our hypothesis, we did not find a significant relationship between infertility or its subtypes and positive status based on DC screening for cervical neoplasia.

An old 1983 cross sectional study in Norway (the only published study identified to have examined a similar relationship) compared the presence of cervical neoplasia among women with tubal factor infertility and women with unclassified infertility (16). The study found that the rate of cervical neoplasia was much higher in the tubal factor infertility group. Unfortunately, the study 
Table 2. DC Results and Other Characteristics of Women Seeking Care for Infertility and Women Seeking Cervical Cancer Screening (Noninfertility Group)

\begin{tabular}{|c|c|c|c|}
\hline \multirow{2}{*}{ Characteristic } & Non-infertility (1836) & Infertility (292) & \multirow{2}{*}{$P$ Value } \\
\hline & No. (\%) & No. (\%) & \\
\hline$D C$ result $(n=2108)$ & & & 0.31 \\
\hline Positive & $248(13.6)$ & 46 (15.9) & \\
\hline Negative (includes Inadequate /Uncertain) & $1570(86.4)$ & $244(84.1)$ & \\
\hline Age group $(n=2124)$ & & & $<0.0001$ \\
\hline $15-24$ & $302(16.5)$ & $74(25.4)$ & \\
\hline $25-34$ & $872(47.6)$ & $164(56.4)$ & \\
\hline $35+$ & $659(36.0)$ & $53(18.2)$ & \\
\hline Years of school $(n=2128)$ & & & $<0.0001$ \\
\hline $0-7$ & $309(16.8)$ & $77(26.4)$ & \\
\hline 8-14 & $376(20.5)$ & $124(42.5)$ & \\
\hline $15+$ & $290(15.8)$ & $64(21.9)$ & \\
\hline Unknown & 861 (46.9) & $27(9.3)$ & \\
\hline Marital status ( $n=2107)$ & & & $<0.0001$ \\
\hline Married & $1231(67.8)$ & $248(85.5)$ & \\
\hline Single, not living with a man & $463(25.5)$ & $38(13.1)$ & \\
\hline Divorced/Separated/Widowed & $123(6.8)$ & $4(1.4)$ & \\
\hline Lifetime partners $(n=2128)$ & & & $<0.0001$ \\
\hline 0-2 partners & $337(18.4)$ & $68(23.3)$ & \\
\hline 3-5 partners & $393(21.4)$ & $96(32.9)$ & \\
\hline $6+$ partners & $77(4.2)$ & $25(8.6)$ & \\
\hline Unknown & $1029(56.1)$ & $103(35.3)$ & \\
\hline Current STI (n=2127) & & & 0.77 \\
\hline cervicitis/pelvic inflammation disease /trichomonas vaginalis & $135(7.4)$ & $20(6.9)$ & \\
\hline Age at first sex $(n=1513)$ & & & 0.09 \\
\hline 0-14 & $92(7.1)$ & $21(10.0)$ & \\
\hline $15-18$ & 727 (55.8) & 125 (59.5) & \\
\hline $19+$ & $484(37.2)$ & $64(30.5)$ & \\
\hline Forced sex (n=1953) & & & 0.4 \\
\hline Yes & $107(6.3)$ & $20(7.7)$ & \\
\hline Sex for money $(n=1941)$ & & & 0.69 \\
\hline Yes & $27(1.6)$ & $5(2.0)$ & \\
\hline
\end{tabular}

Note: The numbers in the tables may differ from the total number of participants because there are missing values for each variable, reason why the total $\mathrm{N}$ for each variable was provided.

did not provide $\mathrm{p}$ values for statistical significance. Tubal infertility is usually a sequelae of STI (1) and therefore it is not surprising that cervical neoplasia was higher in this group.

It has been well documented that STIs such as Chlamydia leads to infertility. Though the mechanism is not yet understood, recent studies are showing that HPV is associated with altered fertility. In women, HPV is capable of causing difficulty in conception and abortions through reduction of endometrial implantation of throphoblastic cells $(17,18)$. In males, HPV is associated with altered sperm motility $(17,19,20)$. With these in mind, one would have expected a strong association between cervical neoplasia and female infertility in our study. However, our study was focused on cervical neoplasia and not on infection with HPV.
In one US study of women with infertility, those with fallopian tube occlusions were at higher risk for cervical cancer compared to those with other causes of infertility (21). Contrary to our study, that study was restricted entirely to women with infertility. Besides the finding suggesting no association between infertility and cervical neoplasia, our other findings support the robustness of our study data. For example, we found that infertility was less prevalent in the women that were 35 or older; the majority of women with infertility problems were between the ages of 25-34 (56.4\%). This finding is similar to findings from a study conducted in Gabon in which the age group 2029 consulted most for infertility with rates of $40.9 \%$ and $52.2 \%$ in rural and semi urban areas respectively (22). The younger age group corresponds to the age group in which conception is most desired. In our population, both 
Table 3. Crude and Adjusted Relative Risk of Infertility

\begin{tabular}{|c|c|c|}
\hline \multirow{2}{*}{ Characteristic } & Crude & Adjusted \\
\hline & RR (95\% Cl) & RR (95\% Cl) \\
\hline \multicolumn{3}{|l|}{ DC results } \\
\hline Positive & $1.16(0.87-1.55)$ & $0.91(0.62-1.20)$ \\
\hline Negative $^{a}$ & 1 (ref) & 1 (ref) \\
\hline \multicolumn{3}{|l|}{ Age group } \\
\hline $15-24$ & $1.24(0.97-1.59)$ & $1.12(0.85-1.40)$ \\
\hline $25-34$ & 1 (ref) & 1 (ref) \\
\hline $35+$ & $0.47(0.35-0.63)$ & $0.53(0.37-0.70)^{a}$ \\
\hline \multicolumn{3}{|l|}{ Years of school } \\
\hline $0-7$ & $0.80(0.63-1.03)$ & $0.80(0.58-1.01)$ \\
\hline $8-14$ & 1 (ref) & 1 (ref) \\
\hline $15+$ & $0.73(0.56-0.95)$ & $0.82(0.58-1.06)$ \\
\hline Unknown & $0.12(0.08-0.18)$ & $0.11(0.04-0.19)^{\mathrm{a}}$ \\
\hline \multicolumn{3}{|l|}{ Marital status } \\
\hline Married & 1 (ref) & 1 (ref) \\
\hline $\begin{array}{l}\text { Single (Not living with } \\
\text { a man), Divorced/ } \\
\text { Separated/Widowed }\end{array}$ & $0.40(0.29-0.55)$ & $0.39(0.25-0.53)^{a}$ \\
\hline \multicolumn{3}{|l|}{ Lifetime partners } \\
\hline $0-2$ partners & $0.86(0.65-1.13)$ & $0.82(0.60-1.04)$ \\
\hline 3-5 partners & 1 (ref) & 1 (ref) \\
\hline $6+$ partners & $1.25(0.85-1.83)$ & $1.18(0.78-1.58)$ \\
\hline Unknown & $0.46(0.36-0.60)$ & $1.15(0.79-1.50)$ \\
\hline \multicolumn{3}{|l|}{ Current STI } \\
\hline Yes & $0.94(0.61-1.43)$ & $1.02(0.61-1.43)$ \\
\hline \multicolumn{3}{|l|}{ Age at first sex } \\
\hline $0-14$ & $1.27(0.83-1.93)$ & $1.10(0.72-1.48)$ \\
\hline $15-18$ & 1 (ref) & 1 (ref) \\
\hline $19+$ & $0.80(0.60-1.05)$ & $0.87(0.66-1.09)$ \\
\hline \multicolumn{3}{|l|}{ Forced sex } \\
\hline Yes & $1.20(0.79-1.82)$ & $0.89(0.49-1.28)$ \\
\hline
\end{tabular}

${ }^{\mathrm{b}}$ Significant predictors in multivariable analysis.

Note: The numbers in the tables may differ from the total number of participants because there are missing values for each variable, reason why the total $\mathrm{N}$ for each variable was provided.

primary and secondary infertility were significantly less prevalent in those who were of older age and those who were unmarried. This could be attributed to less interest in becoming pregnant among women over 35 years of age and among unmarried women, hence less likelihood that these women would present to clinic with complaints of infertility. In general, women with and without positive DC findings did not significantly differ in any of the covariables examined.

A major strength of this study is that it addresses a relationship that only very few published studies have investigated. In addition, we have a relatively large sample size with information and adjustment for several covariables. The study also had a number of limitations. It is a cross-sectional design since infertility and DC findings were ascertained at approximately the same point in time. Thus, the study results although suggestive, cannot definitively allocate or refute a causal relationship. Also, the fertility outcome information was driven primarily by women seeking infertility care on their own volition. It is possible that some subjects in the non-infertility group may have had infertility but just did not consult for infertility, creating a potential bias towards the null in our study.

\section{Conclusions and Implications}

This study explored the relationship between cervical neoplasia and infertility. Participants were women attending a women's health program clinic providing screening for cervical cancer and infertility workups. Overall, infertility did not appear to be associated with cervical neoplasia although contamination in the noninfertility group may bias results towards the null. Future studies should avoid any such contamination, use a cohort or other designs that clearly delineate the temporal relationship between cervical neoplasia and infertility, and importantly, investigate cervical neoplasia using other screening tests or modalities such as Pap smear or HPV typing.

\section{Conflict of Interests}

None of the authors have any specific conflict of interest to state.

\section{Ethical Issues}

This specific analysis received institutional review board (IRB) approval from the CBCHS IRB and waiver of IRB from UAB IRB for de-identified data analysis.

\section{Financial Support}

This study did not receive any specific funding from grant agencies in the public, commercial, or charitable sectors.

\section{References}

1. Centers for Disease Control and Prevention. Sexually Transmitted Diseases Treatment Guidelines. Morbidity and Mortality Weekly Report. 2015 64(3). https://www.cdc.gov/ std/tg2015/tg-2015-print.pdf.

2. Stekler J, Bachmann L, Brotman RM, et al. Concurrent sexually transmitted infections (STIs) in sex partners of patients with selected STIs: implications for patientdelivered partner therapy. Clin Infect Dis. 2005;40(6):78793.

3. Griffiths V, Cheung WH, Carlin EM, Ahmed-Jushuf I. Incidence of concurrent sexually transmitted infections in patients with genital warts. Int J STD AIDS. 2006;17(6):413414. doi: 10.1258/095646206777323328

4. Ferlay J, Soerjomataram I, Ervik M, et al. GLOBOCAN 2012 v1.0, Cancer Incidence and Mortality Worldwide: IARC Cancer Base No. 11. Lyon, France: International Agency for Research on Cancer; 2013. Available from: http://globocan.iarc.fr.

5. World Health Organization. Projections of mortality and burden of disease, World Health Organization. 2004 - 2030.

6. WHO. Comprehensive Cervical Cancer Control. http:// 
www.who.int/reproductivehealth/publications/cancers/ cervical-cancer-guide/en/\%5Cnwww.who.int. Published December 2014.

7. Wright TC, Cox TJ. Cervical Cancer: Epidemiology and Etiology. In: Mayeaux EJ, Cox JT, eds. Modern Colposcopy: Textbook and Atlas. 3rd ed. Philadelphia: Wolters Kluwer/ Lippincott Williams \& Wilkins Health; 2014:61-73.

8. WHO. Guidelines for screening and treatment of precancerous lesions for cervical cancer prevention: supplemental material: GRADE evidence-torecommendation tables and evidence profiles for each recommendation. Geneva: WHO; 2013.

9. Zegers-Hochschild F, Adamson GD, de Mouzon J, et al. International Committee for Monitoring Assisted Reproductive Technology (ICMART) and the World Health Organization (WHO) revised glossary of ART terminology, 2009. Fertil Steril. 2009;92(5):1520-4. doi:10.1016/j. fertnstert.2009.09.009

10. Van de Poel S, Prevalence of Infertility; Focal Point on infertility. On behalf of WHO/RHR and RHP. http:// www.gfmer.ch/infertility/pdf/Prevalence-infertilityVanderpoel-2012.pdf.

11. Cowden AL. Causes of Human Infertility. https://www. texastechphysicians.com/lubbock/obgyn/documents/ Causes_of_Infertility.pdf. Published 2010.

12. Ghadjar P, Budach V, Köhler C, Jantke A, Marnitz S. Modern radiation therapy and potential fertility preservation strategies in patients with cervical cancer undergoing chemoradiation. Radiat Oncol. 2015;10(1):1-6.

13. Chhabra S, Kutchi I. Fertility Preservation in Gynecological Cancers. Clinical Medicine Insights: Reproductive Health. 2013;7:49-59

14. Manga S, Parham G, Benjamin N, et al. Cervical Cancer Screening in Cameroon. J Low Genit Tract Dis.
2015;19(4):288-94.

15. DeGregorio GA, Bradford LS, Manga S, et al. Prevalence, predictors, and same day treatment of positive VIA enhanced by digital cervicography and histopathology results in a cervical cancer prevention program in Cameroon. PLoS ONE 11(6): e0157319. doi:10.1371/journal.pone.0157319

16. Holst N, Abyholm T. Precancerous lesions of the cervix uteri in infertile women. Br Med J. 1983;287:1019-1020.

17. Pereira N, Kucharczyk KM, Estes JL, et al. Human papillomavirus infection, infertility, and assisted reproductive outcomes. J Pathog. 2015;2015:578423. doi: $10.1155 / 2015 / 578423$.

18. Noventa M, Andrisani A, Gizzo S, Nardelli GB, Ambrosini G. Is it time to shift the attention on early stages embryo development to avoid inconclusive evidence on HPVrelated infertility: Debate and proposal. Reprod Biol Endocrinol. 2014;12(1):2-5.

19. Foresta C, Patassini C, Bertoldo A, et al. Mechanism of human papillomavirus binding to human spermatozoa and fertilizing ability of infected spermatozoa. PLoS One. 2011;6(3):e15036. doi:10.1371/journal.pone.0015036.

20. Gizzo S, Ferrari B, Noventa M, Ferrari E, Patrelli TS, Gangemi M, et al. Male and couple fertility impairment due to HPV-DNA sperm infection: Update on molecular mechanism and clinical impact - Systematic review. Biomed Res Int. 2014;2014:20-2

21. Rossing MA, Daling JR, Weiss NS, Moore DE, Self SG. In situ and invasive cervical carcinoma in a cohort of infertile women. Fertil Steril. 1996;65(1):19-22. doi:10.1016/S00150282(16)58021-2

22. Schrijvers D, Dupont A. Meheus A. Prevalence and type of infertility in Gabon. Ann Soc Belge Med Trop. 1991;71:317323.

(C) 2018 The Author (s); This is an open-access article distributed under the terms of the Creative Commons Attribution License (http://creativecommons.org/licenses/by/4.0), which permits unrestricted use, distribution, and reproduction in any medium, provided the original work is properly cited. 